\title{
OPTIMAL BIDDING ALGORITHMS AGAINST CHEATING IN MULTIPLE-OBJECT AUCTIONS*
}

\author{
MING-YANG $\mathrm{KAO}^{\dagger}$, JUNFENG $\mathrm{QI}^{\ddagger}$, AND LEI TAN $\S$
}

\begin{abstract}
This paper studies some basic problems in a multiple-object auction model using methodologies from theoretical computer science. We are especially concerned with situations where an adversary bidder knows the bidding algorithms of all the other bidders. In the two-bidder case, we derive an optimal randomized bidding algorithm, by which the disadvantaged bidder can procure at least half of the auction objects despite the adversary's a priori knowledge of his algorithm. In the general $k$-bidder case, if the number of objects is a multiple of $k$, an optimal randomized bidding algorithm is found. If the $k-1$ disadvantaged bidders employ that same algorithm, each of them can obtain at least $1 / k$ of the objects regardless of the bidding algorithm the adversary uses. These two algorithms are based on closed-form solutions to certain multivariate probability distributions. In situations where a closed-form solution cannot be obtained, we study a restricted class of bidding algorithms as an approximation to desired optimal algorithms.
\end{abstract}

Key words. auction theory, bidding algorithms, electronic commerce, automated negotiation mechanisms, software agents, market-based control

AMS subject classifications. 05A99, 60C05, 68R05, 90A09, 90A12, 90D10, 90D13

1. Introduction. This paper investigates some basic problems in auction theory. Broadly speaking, an auction is a market mechanism with explicit or implicit rules for allocating resources and determining prices on the basis of bids from market participants 进, 10, 12, 18. Auctions are frequently used to price various types of assets. For instance, the U.S. Treasury raises funds by auctioning T-bonds and T-notes, while the Department of the Interior sells mineral rights on federally owned properties via auction. Economists are interested in auctions as an efficient way to price and allocate goods which have no standard market value. Auctions are believed to be the simplest and most familiar means of price determination for multilateral trading without intermediary market makers [10, 12, 18].

In typical auctions, there are one seller and a group of competing buyers who bid to possess the auction objects. Procurements describe situations in which a single buyer wishes to purchase objects from a set of potential suppliers. There are four basic forms of auctions in use [10, 12, 14. In an English auction or ascending bid auction, the price of an object is successively raised until only one bidder remains and wins the object. In a Dutch auction, which is the converse of an English auction, an initial high price is subsequently lowered until a bidder accepts the current price. In a first-price sealed-bid auction, potential buyers submit sealed bids for an object. The highest bidder is awarded the object and pays the amount of his bid. In a second-price sealed-bid auction, the highest bidder wins the object but pays a price equal to the second-highest bid. While there are many other forms of auctions, these four are of the greatest interest.

Previous literature on auction theory mainly studied bidding behavior under the assumption that the objective of bidders is to maximize expected profits in absence

\footnotetext{
*A preliminary version appeared in Proceedings of the 3rd Annual International Computing and Combinatorics Conference, pages 192-201. 1997.

${ }^{\dagger}$ Department of Computer Science, Yale University, New Haven, CT 06520, kao-mingyang@cs.yale.edu. Research supported in part by NSF Grant CCR-9531028.

¥Department of Economics, Duke University, Durham, North Carolina 27708, qijf@econ.duke.edu.

$\S$ Department of Computer Science, Duke University, Durham, North Carolina 27708, lei@cs.duke.edu.
} 
of any budget constraints. Such work concentrates on the allocation of a single object to one of many bidders. Each bidder has a valuation, which is his estimate of the value of the object. In the independent private valuation (IPV) model, each bidder knows his valuation for the object ex ante. Each bidder's valuation is assumed to be drawn independently from the same probability distribution. In the common value (CV) model, it is assumed that bidders obtain imperfect estimates of the value of the object. The bidders all assign the same value to the object ex post. Both models are well studied in auction theory 13, 14, 15, 16.

Very little work in computer science has been conducted on problems related to auctions. Neither auction mechanisms nor bidding algorithms have been formally studied. Nevertheless, computer scientists have realized the importance of auctions as an efficient method of resource allocation [4]. Gagliano et al. applied auction techniques to the allocation of decentralized network resources [9]. Yang et al. proposed an auction-based scheme in which task and resource allocations are determined through negotiations among system entities [19].

Our work investigates some basic issues in the context of automated negotiation mechanisms which are emerging in electronic commerce and other applications of software agents for resource allocation. To maximize transaction volume and speed, we focus on the auction of several objects in parallel and propose a multiple-object auction model. This model further differs from the IPV and CV models in several significant ways. In this model, each bidder faces a binding budget constraint which is identical to all the bidders. Such constraints can be used to enforce fairness of some form when their compliance is verifiable. In electronic transaction environments, security has been a major concern. Our model explicitly considers situations where electronically transmitted information about bids may be legitimately or illegitimately revealed against the wishes of their bidders. In contrast, the IPV and CV models assume that no bidder has an informational advantage on bids or bidding algorithms over other bidders [8].

The assumptions of our model are specified as follows.

- There are a total of $k$ bidders, $\mathcal{B}_{1}, \mathcal{B}_{2}, \ldots, \mathcal{B}_{k}$, each of whom has the same total resource to devote toward winning objects. We normalize this amount to be 1 . Assume that $k \geq 2$.

- A total of $n$ objects are auctioned. Assume that $n \geq k$. Each bidder's goal is to maximize the number of objects he wins. The objects are therefore of equal value to a bidder.

- Each bidder submits a sequence of $n$ bids simultaneously for the $n$ objects. Each object is won by the highest bidder at the price of his bid. If $m$ bidders submit the same highest bid for an object, each wins the object with probability $1 / m$. (Remark: The results of our bidding algorithms in $\$ 2$ and $\$ 3$ are not affected by the specific tie-breaking rules that are used.) For technical reasons, no zero bid is allowed. (Remark: This restriction is only used in \$4.)

- Some bidders may know the bidding algorithms of others. The information structure can be characterized by a directed graph in which an arc from a bidder $\mathcal{B}_{i}$ to another bidder $\mathcal{B}_{j}$ means that $\mathcal{B}_{i}$ knows $\mathcal{B}_{j}$ 's algorithm. For instance, in Figure 1.1, $\mathcal{B}_{4}$ knows the algorithms of $\mathcal{B}_{1}$ and $\mathcal{B}_{2} ; \mathcal{B}_{3}$ knows $\mathcal{B}_{4}$ 's; $\mathcal{B}_{2}$ knows $\mathcal{B}_{3}$ 's; $\mathcal{B}_{1}$ knows only his own. The bidders all compete noncooperatively. We assume that each bidder knows the number of bidders and that of objects.

We analyze the performance of a number of bidding algorithms with which bidders 


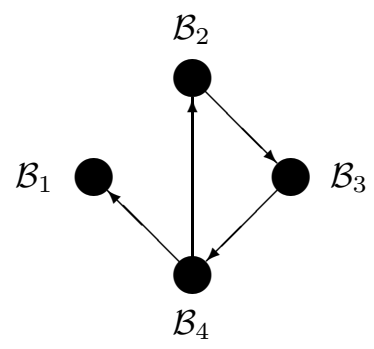

FIG. 1.1. A graph of information structure

can assign their bids. Almost all the bidding algorithms in this paper are randomized ones. We first study the case of two bidders, i.e., $k=2$, and then extend the results to the case of multiple bidders. In the two-bidder case, let $\mathcal{B}$ and $\mathcal{A}$ denote the bidders. We assume that $\mathcal{A}$ knows $\mathcal{B}$ 's bidding algorithm, while $\mathcal{B}$ does not know $\mathcal{A}$ 's, i.e., $\mathcal{B}$ is a disadvantaged bidder and $\mathcal{A}$ an adversary. Here, $\mathcal{A}$ is an oblivious adversary, because although $\mathcal{A}$ knows $\mathcal{B}$ 's bidding algorithm, he does not know the outcome of the random choices that $\mathcal{B}$ makes. We give an optimal randomized bidding algorithm for $\mathcal{B}$ by which he can procure at least one half of the objects despite $\mathcal{A}$ 's a priori knowledge of his bidding algorithm. The main difficulty with obtaining this optimal bidding algorithm is finding a closed-form solution to a desired multivariate probability distribution [1, 5, 6, 11, 17.

We next study the case where there are more than two bidders, and an adversary bidder knows the bidding algorithms of all the others. If the number of objects is a multiple of the number of bidders, an optimal randomized bidding algorithm is found. If all the disadvantaged bidders employ that same bidding algorithm, each of them can obtain at least $1 / k$ of the objects regardless of the bidding algorithm the adversary uses. This bidding algorithm is also based on a closed-form solution to a desired multivariate probability distribution.

When the number of objects is not a multiple of the number of bidders, a closedform solution of a desired probability distribution cannot be obtained. Motivated by this, we study a class of bidding algorithms to approximate desired optimal algorithms. A bidding algorithm in this class computes an initial sequence of bids, and the actual bid sequence is a random permutation of the initial sequence.

Section 2 describes the optimal bidding algorithm for the disadvantaged bidder in the two-bidder case. In $\$ 3$, the optimal randomized bidding algorithm from $\$ 2$ is generalized for the multiple-bidder case. In 细, a class of bidding algorithms are introduced to approximate desired optimal algorithms when a closed-form solution cannot be determined. Section 5 concludes the paper.

For brevity, let $W\left(\mathcal{B}_{i}\right)$ denote the expected number of objects that $\mathcal{B}_{i}$ wins with a bidding algorithm that is explicitly or implicitly specified.

2. The Two-Bidder Case. This section studies the two-bidder case. We assume that $\mathcal{A}$ knows $\mathcal{B}$ 's bidding algorithm, while $\mathcal{B}$ does not know $\mathcal{A}$ 's. We give an optimal randomized bidding algorithm for $\mathcal{B}$ such that $W(\mathcal{B})=n / 2$ despite $\mathcal{A}$ 's informational advantage. Since this problem is a zero-sum game, this bound of $n / 2$ would be straightforward if von Neumann's min-max theorem were applicable. However, our problem has an infinite pure strategy space, and it is not immediately clear that the 
min-max theorem is applicable [2, 3, 7, 8, 18.

2.1. $\mathcal{B}$ 's Optimal Bidding Algorithm. The following lemma gives an upper bound for the expected number of objects $\mathcal{B}$ can win.

LEMma 2.1. $W(\mathcal{B}) \leq \frac{n}{2}$.

Proof. Since $\mathcal{A}$ knows $\mathcal{B}$ 's bidding algorithm, $\mathcal{A}$ can perform at least as well as $\mathcal{B}$ by employing the same algorithm. Then this lemma follows from the fact that our auction is a zero-sum game. $\mathrm{Q}$

Lemma 2.2 describes the marginals of a desired multivariate probability distribution with which $\mathcal{B}$ can form an optimal bidding algorithm.

Lemma 2.2. Assume that $\mathcal{B}$ draws his bid sequence $b_{1}, b_{2}, \ldots, b_{n}$ from an $n$ dimensional probability distribution such that each $b_{i}$ has the same marginal probability distribution $F_{2}\left(b_{i}\right)$, where

$$
F_{2}\left(b_{i}\right)= \begin{cases}\frac{n}{2} \cdot b_{i} & b_{i} \in\left[0, \frac{2}{n}\right] \\ 1 & b_{i} \in\left(\frac{2}{n}, 1\right]\end{cases}
$$

subject to $\sum b_{i}=1$. Then, $\mathcal{A}$ 's optimal bidding algorithm wins exactly $n / 2$ objects on average.

Proof. Let $a_{1}, a_{2}, \ldots, a_{n}$ be $\mathcal{A}$ 's optimal bids for the $n$ objects, respectively. $\mathcal{A}$ 's probability of winning the $i$ th object is $F_{2}\left(a_{i}\right)$. Since $\mathcal{B}$ 's bids are within $[0,2 / n]$, it is not to $\mathcal{A}$ 's advantage to bid over $2 / n$. Hence $a_{i} \leq 2 / n$ and $F_{2}\left(a_{i}\right)=\frac{n}{2} a_{i}$. $\mathcal{A}$ 's optimal bids maximize $W(\mathcal{A})$ as follows:

$$
\begin{aligned}
& \max _{\substack{\sum_{0 \leq 1} a_{i}=1, 0 \leq a_{i} \leq \frac{2}{n}}} W(\mathcal{A})=\max _{\substack{\sum_{0} a_{i}=1, 0 \leq a_{i} \leq \frac{2}{n}}} F_{2}\left(a_{1}\right)+F_{2}\left(a_{2}\right)+\cdots+F_{2}\left(a_{n}\right) \\
& =\max _{\substack{\sum_{0 \leq a_{i}=1} a_{i} \\
0}} \frac{n}{2} \cdot\left(a_{1}+\cdots+a_{n}\right)=\frac{n}{2} .
\end{aligned}
$$

Lemma 2.4 systematically constructs a bid sequence for $\mathcal{B}$ which satisfies the conditions given in Lemma 2.2. We define two additional functions for Lemma 2.4. Let

$$
s(v)=\frac{81}{2} \cdot \frac{v}{2-3 v} .
$$

Let $h(x, y, z)$ be the function defined on $\left\{(x, y, z) \mid 0 \leq x, y, z \leq \frac{1}{3}\right\}$ such that

$$
h(x, y, z)=s(|x-y|+|y-z|+|z-x|) .
$$

LEMma 2.3. The function $h(x, y, z)$ is a joint probability density function of $x, y$ and $z$.

Proof. Note that $h(x, y, z) \geq 0$. To show that $h(x, y, z)$ is a joint probability density function, we need only verify that the integral of $h(x, y, z)$ over $\{(x, y, z) \mid 0 \leq$ $\left.x, y, z \leq \frac{1}{3}\right\}$ is 1 . Let

$$
r(x, y)=\int_{0}^{\frac{1}{3}} h(x, y, z) d z .
$$

Consider the case $x \geq y$. Then

$$
\text { if } x \geq y \geq z, \quad h(x, y, z)=s(2(x-z)) \text {; }
$$




$$
\begin{aligned}
& \text { if } x \geq z \geq y, \quad h(x, y, z)=s(2(x-y)) ; \\
& \text { if } z \geq x \geq y, \quad h(x, y, z)=s(2(z-y)) .
\end{aligned}
$$

Hence if $x \geq y$,

$$
r(x, y)=\int_{0}^{y} s(2(x-z)) d z+\int_{y}^{x} s(2(x-y)) d z+\int_{x}^{1 / 3} s(2(z-y)) d z,
$$

which equals

$$
\frac{9}{2}\left(2 \ln (1-3(x-y))-\ln (3 y(1-3 x))-\frac{1-6(x-y)}{1-3(x-y)}\right) .
$$

By symmetry, if $y \geq x$,

$$
r(x, y)=\frac{9}{2}\left(2 \ln (1-3(y-x))-\ln (3 x(1-3 y))-\frac{1-6(y-x)}{1-3(y-x)}\right) .
$$

It can be verified that

$$
\int_{0}^{1 / 3} \int_{0}^{1 / 3} r(x, y) d x d y=1
$$

Thus,

$$
\int_{0}^{1 / 3} \int_{0}^{1 / 3} \int_{0}^{1 / 3} h(x, y, z) d x d y d z=1
$$

LEMMA 2.4. $\mathcal{B}$ can use the following procedure to draw his bids $b_{1}, b_{2}, \ldots, b_{n}$ such that $\sum b_{i}=1$ and the marginal probability distribution of each $b_{i}$ is as described by (2.1).

Case 1: $n=2 m$ is even. $\mathcal{B}$ draws $b_{1}$ from the probability distribution $F_{2}$ and sets $b_{i}=b_{1}$ and $b_{m+i}=\frac{2}{n}-b_{1}$ for $i=1, \ldots, m$.

Case 2: $n=2 m+1$ is odd. $\mathcal{B}$ draws $b_{1}$ from $F_{2}$ and then sets $b_{i}=b_{1}$ and $b_{m-1+i}=\frac{2}{n}-b_{1}$ for $i=1, \ldots, m-1$. For the remaining three bids $b_{2 m-1}, b_{2 m}, b_{2 m+1}$, $\mathcal{B}$ draws $(x, y, z)$ according to $h$ in $(2.3)$ and sets

$$
b_{2 m-1}=\frac{3}{n}\left(x-y+\frac{1}{3}\right), \quad b_{2 m}=\frac{3}{n}\left(y-z+\frac{1}{3}\right), \quad b_{2 m+1}=\frac{3}{n}\left(z-x+\frac{1}{3}\right) .
$$

Proof. Note that $\sum_{i=1}^{n} b_{i}=1$, whether $n$ is even or odd.

Case 1. This lemma is correct since if a random variable $X$ is drawn from the uniform probability distribution on $\left[0, \frac{2}{n}\right]$, then $\frac{2}{n}-X$ has the same probability distribution.

Case 2. The proof of Case 1 shows that the marginal probability distribution of each $b_{i}$ is $F_{2}$ for $i=1, \ldots, 2 m-2$. It remains to show that $b_{2 m-1}, b_{2 m}, b_{2 m+1}$ are also distributed the same way. Because these three random variables are symmetric to each other in (2.7), we only discuss $b_{2 m-1}$ in detail. Let $t=x-y+\frac{1}{3}$. Since $x$ and $y$ are defined on $\left[0, \frac{1}{3}\right], t$ is defined on $\left[0, \frac{2}{3}\right]$. We have two cases: $t \in\left[0, \frac{1}{3}\right]$ and 
$t \in\left[\frac{1}{3}, \frac{2}{3}\right]$. The two cases are symmetric, and we discuss only the latter. Let $G(t)$ denote the probability distribution of $t$. Then,

$$
G(t)=1-\iint_{u-v+\frac{1}{3} \geq t, 0 \leq u, v \leq \frac{1}{3}} r(u, v) d v d u=1-\int_{t-1 / 3}^{1 / 3} \int_{0}^{u-t+1 / 3} r(u, v) d v d u .
$$

Since $u \geq u-t+1 / 3, r(u, v)$ can take the form of (2.6), and we can obtain $G(t)=\frac{3}{2} t$. Since $b_{2 m-1}=\frac{3}{n} t, F_{2}$ is the probability distribution of $b_{2 m-1}$. $\mathrm{Q}$

THEOREM 2.5. The bidding algorithm given in Lemma 2.4 is optimal for $\mathcal{B}$ and ensures $\mathcal{B}$ at least $n / 2$ objects in expected terms.

Proof. Lemma 2.1 gives an upper bound for $W(\mathcal{B})$. Lemmas 2.2 and 2.4 give an upper bound for $W(\mathcal{A})$, which in turn gives a matching lower bound for $W(\mathcal{B})$ because $W(\mathcal{B})+W(\mathcal{A})=n$.

2.2. Deriving the Joint Probability Density Function $h(x, y, z)$. The most difficult step of obtaining the function $h$ is guessing that $x, y$ and $z$ appear together as $|x-y|+|y-z|+|z-x|$. It is worthwhile to show the derivation of the function $s$ in (2.2) that gives the joint probability density function $h(x, y, z)$. As in (2.4), let $r(x, y)$ be the probability distribution of $(x, y)$. Also let $t=x-y+\frac{1}{3}$. Since $t=\frac{n}{3} b_{2 m-1}$ needs to be uniformly distributed over $\left[0, \frac{2}{3}\right]$, we need to have

$$
1-\int_{t-1 / 3}^{1 / 3} \int_{0}^{u-t+1 / 3} r(u, v) d v d u=\frac{3}{2} t, \text { for all } t \in\left[\frac{1}{3}, \frac{2}{3}\right],
$$

and

$$
\int_{0}^{t} \int_{u-t+1 / 3}^{1 / 3} r(u, v) d v d u=\frac{3}{2} t, \text { for all } t \in\left[0, \frac{1}{3}\right]
$$

These two cases are symmetric, and we only discuss the case given by 2.8) in detail. For notational simplicity, let

$$
s(2 v)=q(v), \quad \int^{u} q(v) d v=p(u), \quad r_{2}(x, y)=\frac{\partial r(x, y)}{\partial y} .
$$

Differentiating (2.8) with respect to $t$ twice, we obtain

$$
\int_{t-1 / 3}^{1 / 3} r_{2}\left(u, u-t+\frac{1}{3}\right) d u+r\left(t-\frac{1}{3}, 0\right)=0 .
$$

Since $x \geq y$ in (2.8), the following is derived from (2.5):

$$
r_{2}(u, v)=-(u-v) q^{\prime}(u-v)-q\left(\frac{1}{3}-v\right)+q(u-v) .
$$

Then,

$$
\begin{aligned}
& \int_{t-1 / 3}^{1 / 3} r_{2}\left(u, u-t+\frac{1}{3}\right) d u \\
= & -\left(t-\frac{1}{3}\right)\left(\frac{2}{3}-t\right) q^{\prime}\left(t-\frac{1}{3}\right)+p\left(t-\frac{1}{3}\right)-p\left(\frac{1}{3}\right)+q\left(t-\frac{1}{3}\right)\left(\frac{2}{3}-t\right) .
\end{aligned}
$$


We obtain from (2.5),

$$
r\left(t-\frac{1}{3}, 0\right)=\left(t-\frac{1}{3}\right) q\left(t-\frac{1}{3}\right)+p\left(\frac{1}{3}\right)-p\left(t-\frac{1}{3}\right) .
$$

Setting $w=t-\frac{1}{3}$, we can derive the following differential equation from (2.9), 2.10) and (2.11):

$$
w(1-3 w) q^{\prime}(w)=q(w) .
$$

The solution to the differential equation is

$$
q(w)=c \frac{3 w}{1-3 w},
$$

where $c$ is a constant. Therefore

$$
s(v)=c \frac{3 v}{2-3 v} .
$$

Since $h(x, y, z)$ is a probability density function for $(x, y, z), c$ is set to $\frac{27}{2}$ to satisfy

$$
\int_{0}^{1 / 3} \int_{0}^{1 / 3} \int_{0}^{1 / 3} h(x, y, z) d x d y d z=1
$$

3. The Multiple-Bidder Case. This section generalizes the results in $\S 2$ to give an optimal randomized bidding algorithm for the case of multiple bidders. We assume that the bidding algorithms of $k-1$ bidders are known to a single adversary bidder $\mathcal{A}$. If all the $k-1$ disadvantaged bidders employ our bidding algorithm, each of them wins at least a fraction $1 / k$ of the objects regardless of the bidding algorithm the adversary uses.

Lemma 3.1. Assume that each of the $k-1$ disadvantaged bidders independently draws his bid sequence $b_{1}, b_{2}, \ldots, b_{n}$ from an $n$-dimensional probability distribution such that each $b_{i}$ has the same marginal probability distribution $F_{k}\left(b_{i}\right)$, where

$$
F_{k}\left(b_{i}\right)= \begin{cases}\left(\frac{n}{k} \cdot b_{i}\right)^{\frac{1}{k-1}} & \text { if } b_{i} \in\left[0, \frac{k}{n}\right], \\ 1 & \text { if } b_{i} \in\left(\frac{k}{n}, 1\right],\end{cases}
$$

subject to $\sum b_{i}=1$. Then, $W(\mathcal{A})$ is at most $n / k$.

Proof. Let $b_{i, j}$ denote the bid on the $i$-th object of the $j$ th disadvantaged bidder. Let $a_{i}$ be $\mathcal{A}$ 's bid on the $i$-th object. Because the bids of the $k-1$ disadvantaged bidders are within $[0, k / n], \mathcal{A}$ has no incentive to bid over $k / n$. Thus, $a_{i} \leq k / n$, and $F\left(a_{i}\right)=\left(\frac{n}{k} \cdot a_{i}\right)^{\frac{1}{k-1}}$. Since bids from different disadvantaged bidders are independent,

$\operatorname{Prob}\left\{a_{i}\right.$ wins the $i$-th object $\}$

$$
\begin{aligned}
& =\operatorname{Prob}\left\{b_{i, 1} \leq a_{i}\right\} \cdot \operatorname{Prob}\left\{b_{i, 2} \leq a_{i}\right\} \cdots \operatorname{Prob}\left\{b_{i, k-1} \leq a_{i}\right\} \\
& =\left(F_{k}\left(a_{i}\right)\right)^{k-1} \\
& =\frac{n}{k} \cdot a_{i} .
\end{aligned}
$$

From the fact that $\sum a_{i} \leq 1, \mathcal{A}$ wins exactly $n / k$ objects on average.

It appears quite difficult to find a closed-form solution to a joint probability distribution whose marginals are as described by (3.1). 
CONJECTURE 3.2. There exists an n-dimensional joint probability distribution such that its marginal probability distribution of every component is as described by (3.1), while the components from all dimensions sum to 1.

For $k=2$, this conjecture has been proved in $\delta 2$. If $n$ is a multiple of $k$, we prove this conjecture as follows. Let

$$
e\left(b_{1}, b_{2}, \ldots, b_{k}\right)= \begin{cases}\left(b_{1} b_{2} \cdots b_{k}\right)^{\frac{1}{k-1}-1} & b_{1}+b_{2}+\cdots+b_{k}=1, b_{i}>0 \\ 0 & \text { otherwise }\end{cases}
$$

Let

$$
\alpha=\int_{b_{1}+\ldots+b_{k}=1} e\left(b_{1}, b_{2}, \ldots, b_{k}\right) d b_{1} d b_{2} \cdots d b_{k-1}
$$

Normalizing $e$ using $\alpha$, we have

$$
g\left(b_{1}, b_{2}, \ldots, b_{k}\right)= \begin{cases}\frac{\left(b_{1} b_{2} \cdots b_{k}\right)^{\frac{1}{k-1}-1}}{\alpha} & b_{1}+b_{2}+\cdots+b_{k}=1, b_{i}>0 \\ 0 & \text { otherwise }\end{cases}
$$

With this normalization, $g$ is a probability density function of $\left(b_{1}, b_{2}, \ldots, b_{k}\right)$. For example, if $n=k=3$, the probability density function shown in (3.2) is

$$
g\left(b_{1}, b_{2}, b_{3}\right)= \begin{cases}\frac{1}{2 \pi \sqrt{b_{1} b_{2} b_{3}}} & b_{1}+b_{2}+b_{3}=1, b_{i}>0 \\ 0 & \text { otherwise }\end{cases}
$$

The following lemma proves Conjecture 3.2 for the case $n=k$.

LEMMA 3.3. If $n=k$ and the bid sequence $b_{1}, b_{2}, \ldots, b_{n}$ is drawn from the $n$-dimensional joint probability distribution in (3.2), then the marginal probability distribution for each $b_{i}$ is as described by (3.1).

Proof. Because $b_{1}, b_{2}, \ldots, b_{k}$ are symmetric for $g$, we need only show that the probability distribution of $b_{k}$ is as described in (3.1). Let

$$
b_{i}=\left(1-b_{k}\right) u_{k-i}, i=2, \ldots, k-1 \text {. }
$$

Then

$$
d b_{i}=\left(1-b_{k}\right) d u_{k-i}
$$

Let

$$
\alpha^{\prime}=\int_{u_{1}+\ldots+u_{k-1}=1}\left(u_{1} u_{2} \ldots u_{k-1}\right)^{\frac{1}{k-1}-1} d u_{k-2} d u_{k-3} \cdots d u_{1} .
$$

Note that $\alpha=(k-1) \cdot \alpha^{\prime}$. The probability distribution of $b_{k}$ equals

$$
\begin{aligned}
& \int_{\substack{0 \leq w \leq b_{k} \\
b_{1}+b_{2}+\cdots+b_{k-1}+w=1}} g\left(b_{1}, \cdots, b_{k-1}, w\right) d b_{2} \cdots d b_{k-1} d w \\
= & \frac{1}{\alpha} \cdot \int_{0}^{b_{k}} \int_{0}^{1-w} \int_{0}^{1-w-b_{k-1}} \cdots \int_{0}^{1-w-\cdots-b_{3}} \\
= & \frac{1}{\alpha} \cdot \int_{0}^{b_{k}} w^{\frac{1}{k-1}-1} \int_{0}^{1} \int_{0}^{1-u_{1}} \cdots \int_{0}^{1-u_{1}-\cdots u_{k-3}}
\end{aligned}
$$




$$
\begin{aligned}
& \left(u_{1} u_{2} \cdots u_{k-2}\left(1-u_{1}-\cdots-u_{k-2}\right)\right)^{\frac{1}{k-1}-1} d u_{k-2} \cdots d u_{2} d u_{1} d w \\
= & \frac{1}{\alpha} \cdot \int_{0}^{b_{k}} \alpha^{\prime} w^{\frac{1}{k-1}-1} d w \\
= & \frac{\alpha^{\prime}}{\alpha}(k-1) a^{\frac{1}{k-1}} \\
= & F_{k}\left(b_{k}\right) .
\end{aligned}
$$

The following lemma extends Lemma 3.3 to the case $n=k \cdot m$ for some integer.

LEмма 3.4. If $n=k \cdot m$ for some integer $m$, there exists a procedure to generate a bid sequence $b_{1}, b_{2}, \ldots, b_{n}$ such that the probability distribution for each $b_{i}$ can be described by (3.1), and the bids $b_{i}$ sum to 1

Proof. If $m=1$, the lemma is the same as Lemma 3.3. If $m>1$, we divide the objects into $m$ groups of $k$ objects each and employ Lemma 3.3 to obtain bids for the first group. We then set the bids for the other $m-1$ groups to the corresponding bids for the first group. We scale every bid by a factor of $\frac{1}{m}$ so that the bids sum to 1. This gives the desired probability distribution.

THEOREM 3.5. If $n=k \cdot m$ for some integer $m$, and the disadvantaged bidders all employ the bidding algorithm characterized by Lemma 3.4, then each can obtain at least $n / k$ objects in expected terms, which is optimal.

Proof. From Lemmas 3.1 and 3.4 and the fact that our game is a zero-sum game, the $k-1$ disadvantaged bidders win $\frac{k-1}{k} \cdot n$ objects in total. Since they all use the same bidding algorithm, by symmetry, each of them wins $n / k$ objects. This upper bound of $n / k$ is also a lower bound since the adversary can always win at least $n / k$ objects by employing the same bidding algorithm as the disadvantaged bidders.

4. Position-Randomized Bidding Algorithms. In \$3 , an optimal randomized bidding algorithm for the bidders with informational disadvantage is derived for the case where the number of objects is a multiple of that of bidders. This algorithm is based on a closed-form solution to a desired multivariate probability distribution. If $n$ is not a multiple of $k$, a closed-form solution cannot be obtained with our current techniques. Motivated by this, we consider situations where all the bidders are restricted to a class of bidding algorithms called position-randomized bidding algorithms. A position-randomized bidding algorithm consists of two steps. Step 1 deterministically selects an initial sequence of $n$ bids. Step 2 permutes the sequence. The $i$-th element of the final sequence is the actual bid for the $i$-th object. As in $\$ 3$, we assume that all the disadvantaged bidders adopt an identical bid sequence at Step 1 and the same probability distribution at Step 2. A position-randomized bidding algorithm can be considered as an approximation to optimal bidding algorithms desired for resolving Conjecture 3.2 in $\$$.

The next lemma examines how probability distributions chosen at Step 2 affect the expected numbers of objects bidders win.

Lemma 4.1. For a given initial bid sequence $a_{1}, a_{2}, \ldots, a_{n}$ of $\mathcal{A}$ and a given initial bid sequence $b_{1}, b_{2}, \ldots, b_{n}$ of the disadvantaged bidders,

- $W_{1}$ denotes the expected number of objects $\mathcal{A}$ wins using the uniform probability distribution while the disadvantaged bidders may use any arbitrary probability distribution;

- $W_{2}$ denotes the expected number of objects $\mathcal{A}$ wins without permuting his initial bid sequence while the disadvantaged bidders employ the uniform probability distribution; 
- $W_{3}$ denotes the expected number of objects $\mathcal{A}$ wins using any given probability distribution while the disadvantaged bidders employ the uniform probability distribution.

If $a_{1}, a_{2}, \ldots, a_{n}$ are all different from $b_{1}, b_{2}, \ldots, b_{n}$, then $W_{1} \geq W_{2}=W_{3}$.

Proof. For each $a_{i}$,

- $W_{1, i}$ denotes the expected number of objects $a_{i}$ wins if $\mathcal{A}$ uses the uniform probability distribution while the disadvantaged bidders may use any arbitrary probability distribution;

- $W_{2, i}$ denotes the expected number of objects $a_{i}$ wins if $\mathcal{A}$ does not permute his initial bid sequence and the disadvantaged bidders employ the uniform probability distribution;

- $W_{3, i}$ denotes the expected number of objects $a_{i}$ wins if $\mathcal{A}$ uses a given probability distribution and the disadvantaged bidders employ the uniform probability distribution.

Since $W_{j}=W_{j, 1}+\cdots+W_{j, n}$ for $j \in\{1,2,3\}$, it suffices to prove that $W_{1, i} \geq W_{2, i}=$ $W_{3, i}$. Without loss of generality, assume that $b_{1} \leq b_{2} \leq \cdots \leq b_{n}$. Let $p$ be the largest index such that $b_{p}<a_{i}$; if no such $b_{p}$ exists, let $p=0$. Since $a_{i}<b_{j}$ for $j=p+1, \ldots, n$,

$$
W_{2, i}=\left(\frac{p}{n}\right)^{k-1}
$$

To calculate $W_{3, i}$, let $Q_{q, r}$ be the probability that $\mathcal{A}$ places $a_{q}$ on the $r$-th object. Then,

$$
\begin{aligned}
W_{3, i} & =\sum_{r=1}^{n} \operatorname{Prob}\left\{a_{i} \text { wins the } r \text {-th object }\right\} \\
& =\sum_{r=1}^{n} Q_{i, r} \cdot\left(\frac{p}{n}\right)^{k-1}
\end{aligned}
$$

Since $\sum_{r=1}^{n} Q_{i, r}=1$,

$$
W_{2, i}=W_{3, i} .
$$

To calculate $W_{1, i}$, let $P_{q, r}$ be the probability that a disadvantaged bidder places $b_{q}$ on the $r$-th object. Then,

$$
\begin{aligned}
W_{1, i} & =\sum_{r=1}^{n} \frac{1}{n} \cdot \operatorname{Prob}\left\{a_{i} \text { wins the } r \text {-th object }\right\} \\
& =\sum_{r=1}^{n} \frac{1}{n} \cdot\left(P_{1, r}+P_{2, r}+\cdots+P_{p, r}\right)^{k-1} .
\end{aligned}
$$

Since $\sum_{r=1}^{n} P_{q, r}=1$ for each $q$, by Hödel's inequality,

$$
W_{1, i} \geq W_{2, i} .
$$

Since $W_{1} \geq W_{3}$ in Lemm 4.1, the disadvantaged bidders should always use the uniform probability distribution at Step 2 . Since $W_{2}=W_{3}$, we may assume that $\mathcal{A}$ does not permute his initial bid sequence whenever the disadvantaged bidders use the 
uniform probability distribution. We next use Lemma 4.1 to derive a lower bound for the expected number of objects $\mathcal{A}$ can win. Let

$$
\begin{aligned}
\epsilon & =\text { a positive infinitesimal amount; } \\
\beta & =\sum_{i=1}^{n} i^{k-1} \\
c_{i} & =\frac{i^{k-1}}{\beta} \\
E & =\left\{c_{0}, c_{1}, c_{2}, \ldots, c_{n}\right\} \\
D & =\left\{\epsilon, c_{2}+\epsilon, c_{3}+\epsilon, \ldots, c_{n}+\epsilon\right\}
\end{aligned}
$$

Lemma 4.2. $\mathcal{A}$ can win at least $\frac{\beta-1}{n^{k-1}}$ objects on average for any given initial bid sequence and probability distribution employed by the disadvantaged bidders.

Proof. Given an initial bid sequence $b_{1} \leq b_{2} \leq \cdots \leq b_{n}$ of the $k-1$ disadvantaged bidders, $\mathcal{A}$ chooses his initial bid sequence to be $b_{1}-(n-1) \epsilon, b_{2}+\epsilon, \ldots, b_{n}+\epsilon$. Since $\mathcal{A}$ 's bids are different from $b_{1}, b_{2}, \ldots, b_{n}$, in light of Lemma 4.1, we may assume that the disadvantaged bidders permute their bids with the uniform probability distribution. Consequently, the expected number of objects won by $\mathcal{A}$ is as desired.

We next prove a matching upper bound for the expected number of objects $\mathcal{A}$ can win.

LEMma 4.3. If the disadvantaged bidders employ $c_{1}, c_{2}, \ldots, c_{n}$ as their initial bid sequence and permute it with the uniform probability distribution, then $\mathcal{A}$ has an optimal initial bid sequence $a_{1}^{\prime}, a_{2}^{\prime}, \ldots, a_{n}^{\prime}$ such that $a_{i}^{\prime} \in D$ for all $i$.

Proof. Given an optimal initial bid sequence $a_{1}, a_{2}, \ldots, a_{n}$ of $\mathcal{A}$, we show that this sequence can be transformed into a desired sequence $a_{1}^{\prime}, a_{2}^{\prime}, \ldots, a_{n}^{\prime}$ without decreasing $W(\mathcal{A})$. Let $m$ be the number of $\mathcal{A}$ 's bids that are in $E$. There are three cases.

Case 1: $m=0$. For each $a_{i}$, let $a_{i}^{\prime}=c_{j}+\epsilon$ where $j$ is the biggest index such that $c_{j}<a_{i}$. Then the expected number of objects won by $a_{1}^{\prime}, a_{2}^{\prime}, \ldots, a_{n}^{\prime}$ is the same as that of $a_{1}, a_{2}, \ldots, a_{n}$, and the new sequence is as desired.

Case 2: $m=1$. This case is impossible since $\mathcal{A}$ can increase $W(\mathcal{A})$ by decreasing one of his bids outside $E$ by $\epsilon$ and increasing the one that is in $E$ by $\epsilon$.

Case 3: $m \geq 2$. Without loss of generality, let $a_{1}, a_{2}, \ldots, a_{m}$ be $\mathcal{A}$ 's $m$ bids in $E$ in the increasing order. We first decrease $a_{1}$ by $(m-1) \epsilon$ and increase $a_{j}$ by $\epsilon$ for $j=2, \ldots, m$. As shown below, this adjustment never decreases $W(\mathcal{A})$. Then, since $\mathcal{A}$ 's adjusted bids are not in $E$, his new initial bid sequence can be further transformed into a desired sequence as in Case 1. Let $w_{1}$ be the decreased amount of $W(\mathcal{A})$ resulted from decreasing $a_{1}$. Let $w_{j}$ be the increased amount of $W(\mathcal{A})$ resulted from increasing $a_{j}$ for $j=2, \cdots, m$. We need to show that $-w_{1}+w_{2}+\cdots+w_{m} \geq 0$. It suffices to prove that $w_{2}-w_{1} \geq 0$. Let $\#_{p}$ denote the expected number of objects $a_{j}$ wins if $a_{j}=c_{p}$. Then,

$$
\begin{aligned}
\#_{p} & =\sum_{i=0}^{k-1} \frac{1}{i+1} \cdot \operatorname{Prob}\left\{a_{j} \text { ties with } i \text { disadvantaged bidders and beats the others }\right\} \\
& =\sum_{i=0}^{k-1} \frac{1}{i+1}\left(\begin{array}{c}
k-1 \\
i
\end{array}\right)\left(\frac{1}{n}\right)^{i}\left(\frac{p-1}{n}\right)^{k-1-i} \\
& =\sum_{i=0}^{k-1} \frac{1}{i+1}\left(\begin{array}{c}
k-1 \\
i
\end{array}\right) \frac{(p-1)^{k-1-i}}{n^{k-1}}
\end{aligned}
$$




$$
=\left(\frac{p-1}{n}\right)^{k-1} \cdot \frac{1}{k} \cdot\left(\left(\frac{1}{p-1}\right)^{k}-1\right) .
$$

Assume that $a_{1}=c_{q}$ and $a_{2}=c_{r}$. Then, $w_{1}=\#_{q}-\left(\frac{q-1}{n}\right)^{k-1}$ and $w_{2}=\left(\frac{r}{n}\right)^{k-1}-\#_{r}$. Note that $w_{2}$ increases with $r$. Since $q \leq r, w_{2}$ is minimized when $a_{1}=a_{2}$ and thus $q=r$. Consequently,

$$
\begin{aligned}
w_{2}-w_{1} & \geq\left(\frac{q}{n}\right)^{k-1}-\#_{q}-\#_{q}+\left(\frac{q-1}{n}\right)^{k-1} \\
& =\left(\frac{q}{n}\right)^{k-1}+\left(\frac{q-1}{n}\right)^{k-1}-2 \cdot \sum_{i=0}^{k-1} \frac{1}{i+1}\left(\begin{array}{c}
k-1 \\
i
\end{array}\right) \frac{(q-1)^{k-1-i}}{n^{k-1}} \\
& =\left(\frac{q}{n}\right)^{k-1}-\left(\frac{q-1}{n}\right)^{k-1}-2 \cdot \sum_{i=1}^{k-1} \frac{1}{i+1}\left(\begin{array}{c}
k-1 \\
i
\end{array}\right) \frac{(q-1)^{k-1-i}}{n^{k-1}} \\
& =\sum_{i=1}^{k-1}\left(\begin{array}{c}
k-1 \\
i
\end{array}\right)\left(\frac{q-1}{n}\right)^{i}\left(\frac{1}{n}\right)^{k-1-i}-2 \cdot \sum_{i=1}^{k-1} \frac{1}{i+1}\left(\begin{array}{c}
k-1 \\
i
\end{array}\right) \frac{(q-1)^{k-1-i}}{n^{k-1}} \\
& =\sum_{i=1}^{k-1}\left(1-\frac{2}{i+1}\right)\left(\begin{array}{c}
k-1 \\
i
\end{array}\right) \frac{(q-1)^{k-1-i}}{n^{k-1}} \\
\geq & 0 .
\end{aligned}
$$

LEMMA 4.4. If the $k-1$ disadvantaged bidders all employ $c_{1}, c_{2}, \ldots, c_{n}$ as their initial bid sequence and permute it with the uniform probability distribution, then $\mathcal{A}$ can win at most $\frac{\beta-1}{n^{k-1}}$ objects on average.

Proof. From Lemma 4.3, $\mathcal{A}$ has an optimal initial bid sequence $a_{1}^{\prime}, a_{2}^{\prime}, \ldots, a_{n}^{\prime}$, such that for all $j, a_{j}^{\prime} \in D$. If $a_{j}^{\prime}=\epsilon$, then it cannot win any object. If $a_{j}^{\prime}=c_{i}+\epsilon$, then it can win $\left(\frac{i}{n}\right)^{k-1}$ objects on average. The unit price $\mathcal{A}$ pays for these objects is strictly greater than

$$
\frac{\frac{i^{k-1}}{\beta}}{\left(\frac{i}{n}\right)^{k-1}}=\frac{n^{k-1}}{\beta} \text {. }
$$

Since the expected number of objects won by such $a_{j}^{\prime}$ is an integral multiple of $\frac{1}{n^{k-1}}$, $W(\mathcal{A})=m \cdot \frac{1}{n^{k-1}}$ for some integer $m$, and

$$
m \cdot \frac{1}{n^{k-1}} \cdot \frac{n^{k-1}}{\beta}<1
$$

Since $m$ is an integer, $m \leq \beta-1$ and thus $W(\mathcal{A}) \leq \frac{\beta-1}{n^{k-1}}$. $\square$

THEOREM 4.5. If the disadvantaged bidders all employ $c_{1}, c_{2}, \ldots, c_{n}$ as their initial bid sequence and permute it with the uniform probability distribution, then each of them can win at least $1 / k$ of $n-\frac{\beta-1}{n^{k-1}}$ objects on average, which is optimal.

Proof. By Lemma 4.4, $\mathcal{A}$ wins at most $\frac{\beta-1}{n^{k-1}}$ objects on average. By Lemma 4.2, this upper bound is also the lower bound of the expected number of objects $\mathcal{A}$ can win. Then this theorem follows from the fact that our auction is a zero-sum game. 
5. Extensions and Open Problems. This paper leaves several problems unsolved. Section 3 still lacks an optimal randomized bidding algorithm for the disadvantaged bidders when $n$ is not a multiple of $k$. In $\S$ 国, if zero bids are allowed, the initial bid sequence $c_{1}, \ldots, c_{n}$ is no longer optimal for the disadvantaged bidders. In general, if disadvantaged bidders do not use identical bidding algorithms, it is not even clear what an optimal bidding algorithm should mean, especially for a more complicated information structure than discussed in this paper.

Our model can be extended to study sequential bidding. The bidders submit sealed bids for an object. Once that object is sold, the next object is auctioned the same way until all the objects are sold. For the case where $n$ is a multiple of $k$, an optimal sequential bidding algorithm is described in the following lemma.

LEMMA 5.1. If $n$ is a multiple of $k$ and the objects are auctioned sequentially, then a bidder can obtain $n / k$ objects by bidding $k / n$ on every object until his budget is exhausted.

Proof. Assume that $\mathcal{B}_{i}$ employs this bidding algorithm. From his budget constraint, he wins at most $n / k$ objects. This upper bound is also a lower bound. To prove this claim by contradiction, assume that $\mathcal{B}_{i}$ wins fewer than $n / k$ objects and thus does not exhaust all his budget. Then, the total number of objects won by the other bidders exceeds $\frac{k-1}{k} \cdot n$. Because $n$ is a multiple of $k$ and $\mathcal{B}_{i}$ has not exhaust his budget, every object's winning bid must be at least $k / n$. Therefore, the total of the winning bids of the other bidders exceeds $k-1$. Since this contradicts the budget constraint, $\mathcal{B}_{i}$ can win at least $n / k$ objects.

Our model can also be extended to the case where the objects may have distinct values. In a general setting, the objects are divided into $m$ groups. Let $n_{i}$ denote the number of objects in the $i$-th group, which may be any positive real number. The bidders are asked to submit bids for the $m$ groups simultaneously. Whoever bids the highest for a group obtains all the objects in that group subject to the same tie-breaking rule. An $m$-group auction is equivalent to an auction of $m$ objects with distinct values where $n_{i}$ is the value of the $i$-th group. As before, assume that an adversary bidder $\mathcal{A}$ knows the bidding algorithms of the other $k-1$ bidders, and all those disadvantaged bidders employ the same bidding algorithm.

Lemma 5.2. Assume that each disadvantaged bidder bids $n_{i} \cdot b_{i}$ for the $i$-th group where $b_{1}, b_{2}, \ldots, b_{m}$ are drawn from an $m$-dimensional probability distribution such that the marginal probability distribution of each $b_{i}$ is $F_{k}$ subject to $n_{1} \cdot b_{1}+\cdots+$ $n_{m} \cdot b_{m}=1$. Then the optimal expected number of objects won by $\mathcal{A}$ is $n / k$.

Proof. Let $n_{i} \cdot b_{i, j}$ denote the bid on the $i$-th object by the $j$-th disadvantaged bidder. Let $n_{i} \cdot a_{i}$ be $\mathcal{A}$ 's bid on the $i$-th object. Because $b_{1}, b_{2}, \ldots, b_{m} \in[0, k / n], \mathcal{A}$ has no incentive to set $a_{i}$ greater than $k / n$. Thus, $a_{i} \leq k / n$ and $F_{k}\left(a_{i}\right)=\left(\frac{n}{k} \cdot a_{i}\right)^{\frac{1}{k-1}}$. Since bids from different disadvantaged bidders are independent,

$$
\begin{aligned}
& \operatorname{Prob}\left\{n_{i} \cdot a_{i} \text { wins the } i \text {-th object }\right\} \\
= & \operatorname{Prob}\left\{b_{i, 1} \leq a_{i}\right\} \cdot \operatorname{Prob}\left\{b_{i, 2} \leq a_{i}\right\} \cdots \operatorname{Prob}\left\{b_{i, k-1} \leq a_{i}\right\} \\
= & \left(F_{k}\left(a_{i}\right)\right)^{k-1} \\
= & \frac{n}{k} \cdot a_{i} .
\end{aligned}
$$

$\mathcal{A}$ maximizes $W(\mathcal{A})$ as follows:

$$
\max _{\substack{\sum_{1} n_{i} \cdot a_{i}=1 \\ 1 \leq a_{i} \leq \frac{k}{n}}} W(\mathcal{A})
$$




$$
\begin{aligned}
& =\underset{\substack{\sum_{1 \leq a_{i} \leq \frac{k}{n}} n_{i} \cdot a_{i}=1 \\
1}}{ } n_{1} \cdot\left(\frac{n}{k} \cdot a_{1}\right)+n_{2} \cdot\left(\frac{n}{k} \cdot a_{2}\right)+\cdots+n_{m} \cdot\left(\frac{n}{k} \cdot a_{m}\right) \\
& =\frac{n}{k} .
\end{aligned}
$$

CONJECTURE 5.3. There exists an $m$-dimensional probability distribution for $\left(b_{1}, b_{2}, \ldots, b_{m}\right)$ subject to the constraint $n_{1} \cdot b_{1}+n_{2} \cdot b_{2}+\cdots+n_{m} \cdot b_{m}=1$ such that the marginal probability distribution of each $b_{i}$ is as described by (3.1).

Remark. This conjecture can be reduced to the case $m=2$ or 3 .

We conclude the paper with two research directions. One is to consider general information structures as specified by arbitrary directed graphs. The other is to investigate more general budget constraints beyond the homogeneous one of this paper. It would be of significance to design bidding algorithms that can optimally or approximately achieve game-theoretic equilibria in meaningful combinations of these two directions.

Acknowledgements. We are indebted to Phil Long, Kasturi Varadarajan, and Professor Dennis Yang at the Economics Department of Duke University for very helpful comments. We also wish to thank anonymous referees for contagious enthusiasm towards this work and unusually thoughtful comments and detailed suggestions.

\section{REFERENCES}

[1] V. Benes And J. Stepan, Extremal solutions in the marginal problem, in Advances in Probability Distributions with Given Marginals, 1991, pp. 189-206.

[2] D. Blackwell, An analog of the minimax theorem for vector payoffs, Pacific Journal of Mathematics, 6 (1956), pp. 1-8.

[3] D. Blackwell and M. A. Girshick, Theory of Games and Statistical Decisions, John Wiley \& Sons, 1954.

[4] S. H. Clearwater, ed., Market-Based Control, a Paradigm for Distributed Resource Allocation, World Scientific, River Ridge, New Jersey, 1996.

[5] G. Dall'Aglio, Fréchet classes: The beginnings, in Advances in Probability Distributions with Given Marginals, 1991, pp. 1-12.

[6] M. FrÉchet, Sur les tableaux de corrélation dont les marges sont données, Annales de l'Université de Lyon, Lyon Science, 20 (1951), pp. 13-31.

[7] Y. Freund and R. Schapire, Game theory, on-line prediction and boosting, in Proceedings of the Ninth Annual Conference on Computational Learning Theory, 1996.

[8] D. Fudenberg and J. Tirole, Game Theory, Mit Press, Cambridge, Massachusetts, 1991.

[9] R. A. Gagliano, M. D. Fraser, and M. E. Schaefer, Auction allocation of computing resources, Communications of the ACM, 38 (1995), pp. 88-99.

[10] K. HendRICKS AND H. J. PAARSh, A survey of recent empirical work concerning auctions, Canadian Journal of Economics, 28 (1995), pp. 403-426.

[11] S. Kotz And J. P. Seeger, A new approach to dependence in multivariate distributions, in Advances in Probability Distributions with Given Marginals, 1991, pp. 113-128.

[12] J. McMillan and R. P. McAfee, Auctions and bidding, Journal of Economic Literature, 25 (1987), pp. 699-738.

[13] P. R. Milgrom, Good news and bad news: Representation theorems and applications, Bell Journal of Economics, 12 (1981), pp. 380-391.

[14] P. R. Milgrom and R. J. Weber, A theory of auctions and competitive bidding, Econometrica, 50 (1982), pp. 1089-1122.

[15] R. B. Myerson, Optimal auction design, Mathematics of Operations Research, 6 (1981), pp. $58-73$.

[16] C. Pitchik And A. Schotter, Perfect equilibria in budget-constrained sequential auctions: an experimental study, RAND Journal of Economics, 19 (1988), pp. 363-388.

[17] B. Schweizer, Thirty years of copulas, in Advances in Probability Distributions with Given Marginals, 1991, pp. 13-50. 
[18] R. Wilson, Handbook of Game Theory, Elsevier Science Publishers, 1992.

[19] E. H. Yang, M. M. Barash, And D. M. Upton, Accommodation of priority parts in a distributed computer-controlled manufacturing system with aggregate bidding schemes, in Proceedings of the 2nd Industrial Engineering Research Conference, 1993, pp. 827-831. 